\title{
Design of a dual-channel modelocked fiber laser that avoids multi-pulsing
}

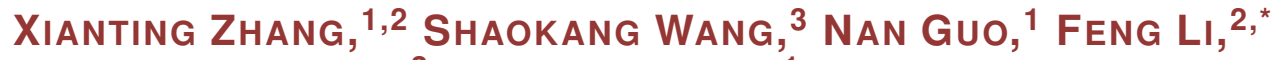 \\ CuRTIS R. MENYUK, ${ }^{3}$ AND P. K. A. WAI ${ }^{1}$ \\ ${ }^{1}$ Photonics Research Centre, Department of Electronic and Information Engineering, \\ The Hong Kong Polytechnic University, Hung Hom, Kowloon, Hong Kong, China \\ ${ }^{2}$ The Hong Kong Polytechnic University Shenzhen Research Institute, Shenzhen 518057, China \\ ${ }^{3}$ Department of Computer Science and Electrical Engineering, University of Maryland, Baltimore County, \\ 1000 Hilltop Circle, Baltimore, MD 21250, USA \\ *enlf@polyu.edu.hk
}

\begin{abstract}
Multi-channel modelocked lasers and their design have attracted much attention. Here, we use the Swift-Hohenberg equation to study dual-channel simultaneous modelocking (DSML) in a fiber laser. When a quartic filter is added to the laser cavity, the stable dual-channel simultaneous modelocking can be obtained for a given filter bandwidth when frequency separation, $\omega_{\mathrm{s}}$, is less than a calculated threshold, $\omega_{\text {th }}$. When $\omega_{\mathrm{s}}>\omega_{\text {th }}$, a multipulsing instability occurs. We use a linear stability analysis to determine the limit that the multi-pulsing instability imposes on DSML, and we propose a cavity design that avoids the multi-pulsing instability.
\end{abstract}

(C) 2019 Optical Society of America under the terms of the OSA Open Access Publishing Agreement

\section{Introduction}

Modelocked fiber lasers have a wide range of applications in many fields including metrology [1], biomedical imaging [2], and communications [3]. During the last three decades, single channel modelocked fiber lasers have been studied intensively both theoretically and experimentally [4-8]. Modelocking in fiber lasers is achieved by the interaction between dispersion, self-phase modulation, and saturable absorption. Saturable absorption can be obtained by using a nonlinear polarization rotation device [9-11] or a semiconductor saturable absorbing mirror [12,13], among other approaches.

Recent advances in areas like fiber optic sensing and optical computing have led to growing interest in multi-channel modelocked laser sources [14-19]. These light sources can generate pulses that are synchronously modelocked with different central wavelengths $[9,11,13-16,20-22]$. Multi-channel modelocking was initially realized by inserting a dual-channel filter inside the laser cavity [23,24]. The dual-channel filter suppresses all but a few longitudinal modes that are not attenuated by the two frequency channels of the filter. The light that propagates in these two filter channels interacts via four wave mixing and eventually becomes phase-locked as a stable modelocked state is achieved. In the time domain, the modelocked waveform has a rapid amplitude oscillation. This rapidly oscillating waveform is a pulse train with a high repetition rate [14].

Starting from noise, multi-channel modelocking can be initiated by generating modelocked pulses in a single channel first, and then increasing the pump power [13]. However, it has been reported that a multi-pulsing instability can occur when the pump power increases [11,22]. This multi-pulsing instability inhibits multi-channel modelocking in lasers with multi-channel filters. In this manuscript, we will investigate the requirement to avoid a multi-pulsing instability as the pump power increases.

There have been computational studies of the multichannel modelocked laser dynamics in which the evolution equations are solved using evolutionary methods in which one starts from an initial condition and the time is then stepped in small increments [25]. This approach is 
computationally inefficient and can be ambiguous because it is always debatable how long the propagation time should be. The computation time approaches infinity when the system is marginally stable. An alternative approach based on dynamical system theory is to find the modelocked solution using root-finding methods as the laser parameters vary and to determine its linear stability using spectral methods. This dynamical approach is computationally efficient and unambiguous [26-28]. Thus, it can be a powerful tool for laser design and optimization.

During the last few decades, single-channel modelocking has been intensively studied using the Haus modelocking equation and its extensions [29-32]. Models that use coupled equations have been proposed to investigate their dynamics $[15,16,33]$. In these models, the laser cavity is described using a set of partial differential equations in which each equation represents the light evolution inside a single filter channel. These models work well, but they do not include the effect of four-wave mixing, which is important to overcome the gain competition among the channels if a homogeneous gain medium is used $[15,16,33]$.

A simple model that describes multi-channel modelocking and includes four-wave mixing is the Swift-Hohenberg equation (SHE) [25,34]. The SHE is an extension of the well-known Haus modelocking equation [25,27,34-38], which is one of the oldest and most widely-used models for modelocked lasers. In this article, we extend the analysis in [34]. We study dual-channel modelocking based on the SHE using both the evolutionary approach and the dynamical approach.

The remainder of this manuscript is organized as follows: In Sec. 2, we will present the SHE. In Sec. 3 we will then obtain the stationary solutions that correspond to the modelocked pulses and study their stability. We will show that when the frequency separation of the modelocked channels increases, the real part of some eigenvalues in the continuous spectrum become positive, leading to the multi-pulsing instability. We computationally study the requirements to achieve the dual-channel simultaneously modelocking state in a fiber laser while avoiding the multi-pulsing instability.

\section{The Swift-Hohenberg equation}

The Swift-Hohenberg equation $[25,35]$ can be written as

$$
\frac{\partial q}{\partial z}=-i \phi q-D_{1} \frac{\partial q}{\partial t}+\frac{g(|q|)}{2} q-\frac{l}{2} q+\frac{i}{2} \frac{\partial^{2} q}{\partial t^{2}}+(i+\delta)|q|^{2} q-\sigma|q|^{4} q+\alpha_{2} \frac{\partial^{2} q}{\partial t^{2}}+\alpha_{4} \frac{\partial^{4} q}{\partial t^{4}},
$$

where $z$ is normalized to the cavity length $L_{\mathrm{c}}$. The complex envelope $q$ and the delayed time $t$ are normalized using

$$
q=A\left(\gamma L_{\mathrm{c}}\right)^{1 / 2}, \quad t=\tau\left(\left|\beta_{2}\right| L_{\mathrm{c}}\right)^{-1 / 2},
$$

in which $A$ is the slowly varying field envelope, $\gamma$ is the Kerr nonlinearity coefficient, $\tau$ is the delayed physical time, and $\beta_{2}$ is the group-velocity dispersion. Here, we use normalized parameters in Eq. (1), in which the coefficients of the group velocity dispersion term and the Kerr nonlinearity term are normalized to 1 . The coefficient $\phi$ is the phase rotation per roundtrip and $D_{1}$ is the coefficient of the first-order chromatic dispersion. The value of $\phi$ is commonly set to zero since it has no effect on the pulse evolution except to induce on overall phase rotation. The value of $D_{1}$ is similarly often set to zero since it can be removed by using retarded time in which the group velocity motion is subtracted.

Mathematically, adding $\phi$ and $D_{1}$ to the model allows one to obtain strictly stationary solutions, which is convenient for the stability analysis [27]. Physically, the phase shift $\phi$ corresponds to the modelocked pulse's phase change in one roundtrip relative to the carrier frequency's linear phase rotation. The quantity $D_{1}$ corresponds to the modelocked pulse's normalized time shift in one roundtrip relative to the linear time shift due to the group velocity motion.

We are modeling a laser with fast saturable absorption and slow saturable gain. The parameters $\delta$ and $\sigma$ are the coeffecients for the cubic and quintic terms, respectively, or the fast saturable 


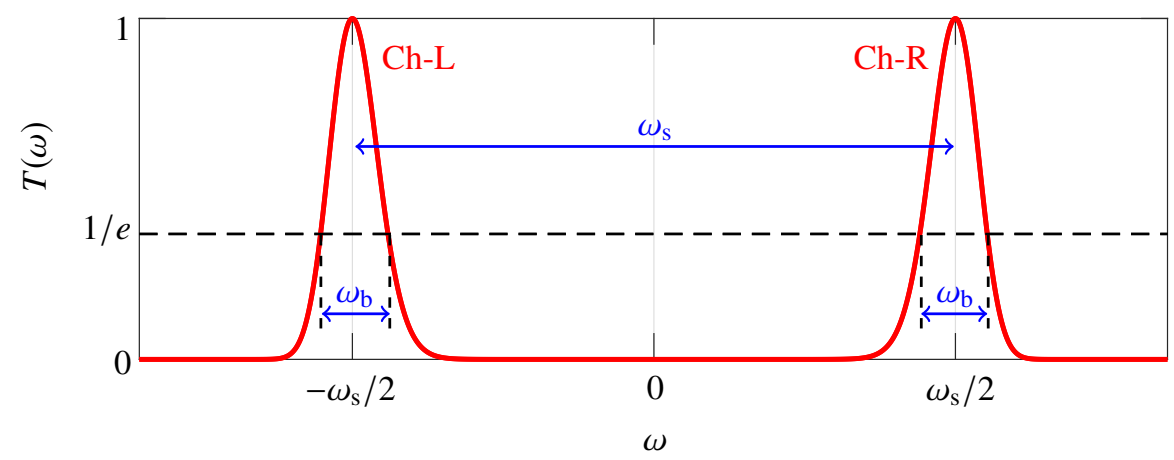

Fig. 1. The filter profile in the Fourier domain and the filter parameters.

absorption [27]. The gain $g(|q|)$ is saturated by the average energy inside the cavity, so that

$$
g(|q|)=g_{0}\left[1+\frac{\int_{0}^{T_{\mathrm{R}}}|q|^{2} \mathrm{~d} t}{E_{\mathrm{sat}}}\right]^{-1},
$$

where $g_{0}$ is the unsaturated gain, $E_{\mathrm{sat}}$ is the saturation energy and $T_{\mathrm{R}}$ is the roundtrip time.

In this paper, we consider a dual-channel intra-cavity filter, and we show an illustration of its frequency profile in Fig. 1. The dual-channel filter's transmission function can be written as $[25,35]$

$$
T(\omega)=\exp \left\{\alpha_{4}\left[\omega^{2}-\left(\omega_{\mathrm{s}} / 2\right)^{2}\right]^{2}\right\},
$$

where $\omega$ is the normalized angular frequency that has been shifted so that the maxima of $T(\omega)$ are symmetric about $\omega=0$, and $\omega_{\mathrm{s}}$ is the frequency offset of the transmission maxima. The parameter $\omega_{\mathrm{s}}$ and $\alpha_{4}$ define the profile of the filter. We set $\alpha_{4}<0$ so that the filter transmission has two maxima at $\omega= \pm \omega_{\mathrm{s}} / 2$. Throughout the paper, we refer to the filter channel at $-\omega_{\mathrm{s}} / 2$ as "Ch-L" and the channel at $\omega_{\mathrm{s}} / 2$ as "Ch-R." For each filter channel, we define the channel bandwidth $\omega_{\mathrm{b}}$ to be the positions where the filter transmission decreases to $1 / e$ of its maximum, as illustrated in Fig. 1. In Eq. (1), we set

$$
\alpha_{2}=-\alpha_{4} \omega_{\mathrm{s}}^{2} / 8
$$

in order for Eq. (1) to be consistent with Eq. (4). Similarly, we set the linear loss coefficient $l$ as

$$
l=l_{\text {cavity }}+\alpha_{4} \omega_{\mathrm{s}}^{4} / 16,
$$

where $l_{\text {cavity }}$ represents the background loss of the laser cavity, excluding the intra-cavity dualchannel filter. In physical units, the corresponding angular frequency separation between $\mathrm{Ch}-\mathrm{L}$ and $\mathrm{Ch}-\mathrm{R}$ can be calculated using

$$
\Omega_{\mathrm{s}}=\left(\left|\beta_{2}\right| L_{\mathrm{c}}\right)^{-1 / 2} \omega_{\mathrm{s}} / 2 .
$$

\section{Results and discussion}

Using Eq. (1), we can find both single- and dual-channel modelocked states when we change the cavity gain and the filter parameters. In this section, we study the modelocked states of Eq. (1) to provide a background for the cavity design of a dual-channel modelocked fiber laser. 


\subsection{Modelocked states}

In this section, we find solutions of the SHE by solving Eqs. (1) and (3) using the split-step Fourier method [36]. We list the parameters that we use in our computation in Table. 1. We set the filter bandwidth $\omega_{\mathrm{b}}=0.3$ and the channel separation $\omega_{\mathrm{s}}=1.20$. Using Eqs. (4) and (5), we obtain the corresponding values of $\alpha_{2}$ and $\alpha_{4}$. According to our normalization, these parameters correspond to a physical filter bandwidth of about $100 \mathrm{GHz}$ when the cavity length $l_{\text {cavity }}$ is $10 \mathrm{~m}$ and $\beta_{2}$ is $-20 \mathrm{ps}^{2} / \mathrm{km}$, which are typical values for fiber lasers. We use an initial condition of white Gaussian noise for $q$ with zero mean and a variance nearly equal to $10^{-6}$. When we set $E_{\text {sat }}=0.50$, the system evolves to the modelocked state that exists in Ch-L, as shown in Figs. 2(a) and 2(b). However, we have observed that the same modelocked solution can also appear in Ch-R instead, depending on the initial noise realization.

Table 1. Values of Parameters We Use in Validating the Experimental Results

\begin{tabular}{|c|c|c|c|c|c|c|c|}
\hline Parameter & $g_{0}$ & $l$ & $\delta$ & $\sigma$ & $\omega_{\mathrm{s}}$ & $\alpha_{2}$ & $\alpha_{4}$ \\
\hline Value & 6.9 & 9.43 & 0.4 & 0.4 & 1.20 & 23.76 & -32.92 \\
\hline
\end{tabular}
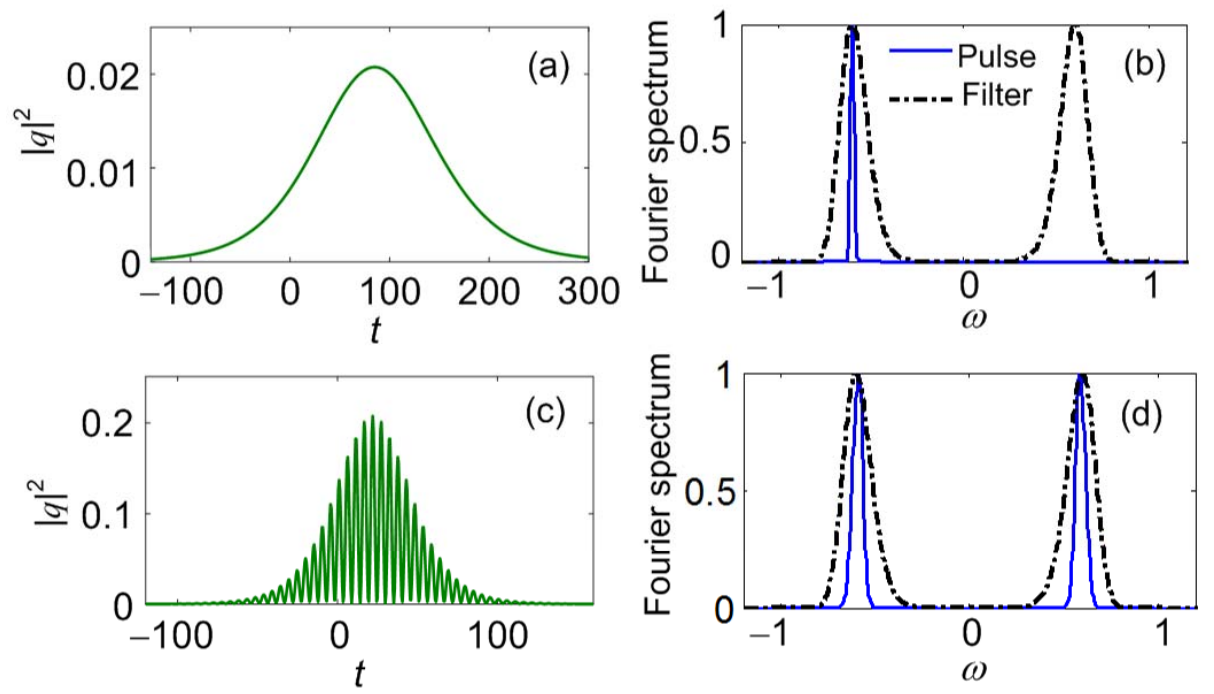

Fig. 2. The temporal profiles and the normalized Fourier spectra of stable modelocked states that we obtain by evolving Eq. (1) when (a)(b) $E_{\mathrm{sat}}=0.50$, and (c)(d) $E_{\mathrm{sat}}=1.00$. The parameters are $\omega_{\mathrm{s}}=1.20$ and $\omega_{\mathrm{b}}=0.30\left(\alpha_{2}=23.76\right.$ and $\left.\alpha_{4}=-32.92\right)$.

If we increase the pump power, the single-channel modelocked pulse can evolve to a dualchannel modelocked state. The action of increasing the pump power in experiments can be modeled by increasing the value of $g_{0}$ and/or $E_{\text {sat }}$ in Eq. (3). In this paper, we set $E_{\text {sat }}=1.00$, which increases the cavity gain compared to the case shown in Figs. 2(a) and 2(b). We show the new modelocked state in Figs. 2(c) and 2(d). In this case, the optical signal exists in both Ch-L and Ch-R, as shown in Fig. 2(d). The phase difference between the signals in these two channels are locked via four-wave mixing, which leads to a visible oscillation in the pulse's amplitude in the time domain. This oscillation occurs because the signals in the two filter channels propagate with the same group velocity, but have different frequencies that beat with each other. This result 
agrees with the experimentally observed profiles of multichannel modelocking [14]. We refer to this modelocked state as the dual-channel modelocked state. These laser sources can be used to generate a high-repetition rate pulse train (or pulse packet) and can potentially be deployed in wavelength-division multiplexed systems [14,20].

The modulation period that we show in Fig. 2(c), which is referred to as the repetition rate of the train of sub-pulses in [14], increases as the separation of the central frequencies of the filters grows [14]. However, dual-channel modelocking ceases to occur when the frequency separation becomes sufficiently large. In Fig. 3, we show the appearance of multipulsing when the pump power increases. In this case, we increased the channel separation by setting $\omega_{\mathrm{s}}=1.60$, and we observed that a multi-pulse state appears when $E_{\text {sat }}$ increases. We obtained two modelocked pulses that have the same peak intensity of 0.13 and a pulse duration of 57.67, which both exist in Ch-L. The multi-pulsing instability prevents the formation of a dual-channel modelocked state.

From the observation in Figs. 2 and 3, we infer that there exists a competition between the dual-channel modelocked state and the multi-pulse state when the cavity gain increases. In the next section, we will use the dynamical approach to find the requirement to avoid the multi-pulsing instability and obtain the dual-channel modelocked state when the gain increases.
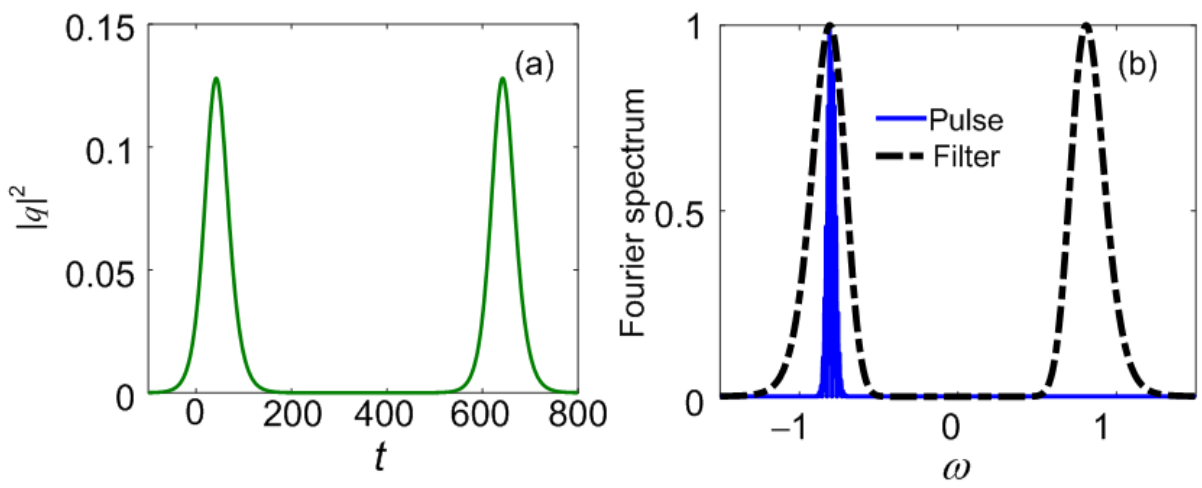

Fig. 3. The temporal profiles and the normalized Fourier spectra of modelocked states that we obtain by solving Eq. (1) when $E_{\mathrm{sat}}=2.50$. We set $\omega_{\mathrm{s}}=1.60$ and $\omega_{\mathrm{b}}=0.30$ $\left(\alpha_{2}=23.04, \alpha_{4}=-17.98\right)$.

\subsection{Stability of the modelocked states}

Here, we briefly describe the dynamical approach,

1. We first find the stationary solution, $q_{0}$, as well as $\phi_{0}$, by solving a root-finding problem computationally [27],

$$
\frac{\partial q_{0}(t)}{\partial z}=0
$$

2. We linearize the system equation - in this case, Eq. (1) —around the stationary solution $\left[q_{0}, \phi_{0}\right]$ that we obtained and we evaluate the dynamical spectrum of the linearized operator $[27,39]$. The dynamical spectrum includes all eigenvalues of the linearized system.

3. The stationary solution is stable when no eigenvalues have a positive real part. 
When linearizing a system in which the complex conjugate explicitly appears, as is the case for Eq. (1), we must extend the linearized system, treating the complex conjugate equation independently [40]. For computational convenience, we write both the stationary solution and the system as a combination of their real parts and imaginary parts,

$$
\begin{aligned}
q_{0}(t) & =R_{0}(t)+i I_{0}(t), \\
R(t, z) & =R_{0}(t)+\Delta R(t, z), \\
I(t, z) & =I_{0}(t)+\Delta I(t, z),
\end{aligned}
$$

where $R_{0}$ and $I_{0}$ are the real and imaginary components of the stationary solution $q_{0}$, while $\Delta R$ and $\Delta I$ are the perturbations to $R_{0}$ and $I_{0}$.

In simulations, the time $t$ is discretized as $t_{1}, t_{2}, \cdots, t_{N}$ with an identical spacing of $\Delta t$. The computational time window $T_{w}$ is chosen large enough to avoid any visible impact on plotted results. We have doubled $T_{w}$ and $N$ and did not observe a visible change in our results. As an example, the vector $q_{0}(t)$ becomes $\mathbf{q}_{0}=\left[q_{0}\left(t_{1}\right), q_{0}\left(t_{2}\right), \cdots, q_{0}\left(t_{N}\right)\right]^{T}$.

We substitute Eq. (9) into Eq. (1) and Eq. (3), and we obtain

$$
\frac{\mathrm{d}}{\mathrm{d} z}\left[\begin{array}{c}
\Delta \mathbf{R} \\
\Delta \mathbf{I}
\end{array}\right]=\mathrm{J}\left[\begin{array}{c}
\Delta \mathbf{R} \\
\Delta \mathbf{I}
\end{array}\right]=\left[\begin{array}{ll}
\mathrm{L}_{11} & \mathrm{~L}_{12} \\
\mathrm{~L}_{21} & \mathrm{~L}_{22}
\end{array}\right]\left[\begin{array}{c}
\Delta \mathbf{R} \\
\Delta \mathbf{I}
\end{array}\right],
$$

where $\mathrm{J} \in \mathbb{R}^{2 N \times 2 N}$ is the Jacobian matrix whose entries are defined as

$$
\begin{aligned}
& \mathrm{L}_{11}=-D_{1} \mathrm{D}_{t}+\alpha_{2} \mathrm{D}_{t}^{2}+\alpha_{4} \mathrm{D}_{t}^{4}+\frac{g-l}{2} \mathrm{I}+g_{1} \mathbf{R}_{0} \mathbf{R}_{0}^{T}+\mathrm{P}_{R}, \\
& \mathrm{~L}_{12}=\phi_{0} \mathrm{I}-\frac{1}{2} \mathrm{D}_{t}^{2}+g_{1} \mathbf{R}_{0} \mathbf{I}_{0}^{T}+\mathrm{P}_{I}, \\
& \mathrm{~L}_{21}=-\phi_{0} \mathrm{I}+\frac{1}{2} \mathrm{D}_{t}^{2}+g_{1} \mathbf{I}_{0} \mathbf{R}_{0}^{T}+\mathrm{Q}_{R}, \\
& \mathrm{~L}_{22}=-D_{1} \mathrm{D}_{t}+\alpha_{2} \mathrm{D}_{t}^{2}+\alpha_{4} \mathrm{D}_{t}^{4}+\frac{g-l}{2} \mathrm{I}+g_{1} \mathbf{I}_{0} \mathbf{I}_{0}^{T}+\mathrm{Q}_{I}
\end{aligned}
$$

The operator $\mathrm{D}_{t}^{m}$ is the $m$-th order differential matrix which we obtain by using a spectral differentiation scheme [27]. The gain is given by $g=g\left(\left|q_{0}\right|\right)$, and the coefficient $g_{1}$ is given by

$$
g_{1}=-\frac{g_{0}}{\left[1+\Delta t\left(\mathbf{R}_{\mathbf{0}}^{\mathbf{T}} \mathbf{R}_{\mathbf{0}}+\mathbf{I}_{\mathbf{0}}^{\mathbf{T}} \mathbf{I}_{\mathbf{0}}\right) / E_{\mathrm{sat}}\right]^{2}} \frac{\Delta t}{E_{\mathrm{sat}}} .
$$

The matrices $\mathrm{P}_{R}, \mathrm{P}_{I}, \mathrm{Q}_{I}$, and $\mathrm{Q}_{R}$ are diagonal and are given by:

$$
\begin{aligned}
\mathbf{P}_{R, j j} & =-2 \mathbf{R}_{j} \mathbf{I}_{j}+\delta\left(3 \mathbf{R}_{j}^{2}+\mathbf{I}_{j}^{2}\right)-\sigma\left[\left(\mathbf{R}_{j}^{2}+\mathbf{I}_{j}^{2}\right)^{2}+4 \mathbf{R}_{j}^{2}\left(\mathbf{R}_{j}^{2}+\mathbf{I}_{j}^{2}\right)^{2}\right], \\
\mathbf{P}_{I, j j} & =-\left(\mathbf{R}_{j}^{2}+3 \mathbf{I}_{j}^{2}\right)+2 \delta \mathbf{R}_{j} \mathbf{I}_{j}-4 \sigma \mathbf{R}_{j} \mathbf{I}_{j}\left(\mathbf{R}_{j}^{2}+\mathbf{I}_{j}^{2}\right), \\
\mathbf{Q}_{R, j j} & =-\left(\mathbf{I}_{j}^{2}+3 \mathbf{R}_{j}^{2}\right)+2 \delta \mathbf{R}_{j} \mathbf{I}_{j}-4 \sigma \mathbf{R}_{j} \mathbf{I}_{j}\left(\mathbf{R}_{j}^{2}+\mathbf{I}_{j}^{2}\right), \\
\mathbf{Q}_{I, j j} & =2 \mathbf{R}_{j} \mathbf{I}_{j}+\delta\left(3 \mathbf{I}_{j}^{2}+\mathbf{R}_{j}^{2}\right)-\sigma\left[\left(\mathbf{R}_{j}^{2}+\mathbf{I}_{j}^{2}\right)^{2}+4 \mathbf{R}_{j}^{2}\left(\mathbf{R}_{j}^{2}+\mathbf{I}_{j}^{2}\right)^{2}\right] .
\end{aligned}
$$

In Fig. 4, we show the dynamical spectrum of the single channel modelocked solution in Figs. 2(a) and 2(b). The dynamical spectrum includes branches of continuous eigenvalues and multiple discrete eigenvalues. As shown in Fig. 4, no eigenvalues have a positive real part, and hence the stationary solution is stable. We observe that a pair of complex conjugate eigenvalues, $\lambda_{\mathrm{d}}$ and $\lambda_{\mathrm{d}}^{*}$, are visible near the imaginary axis.

When we increase $E_{\text {sat }}$ from 0.50 to 1.00 , we find that the single-channel modelocked solution continues to exist but becomes unstable, as indicated by the dynamical spectrum that we show 


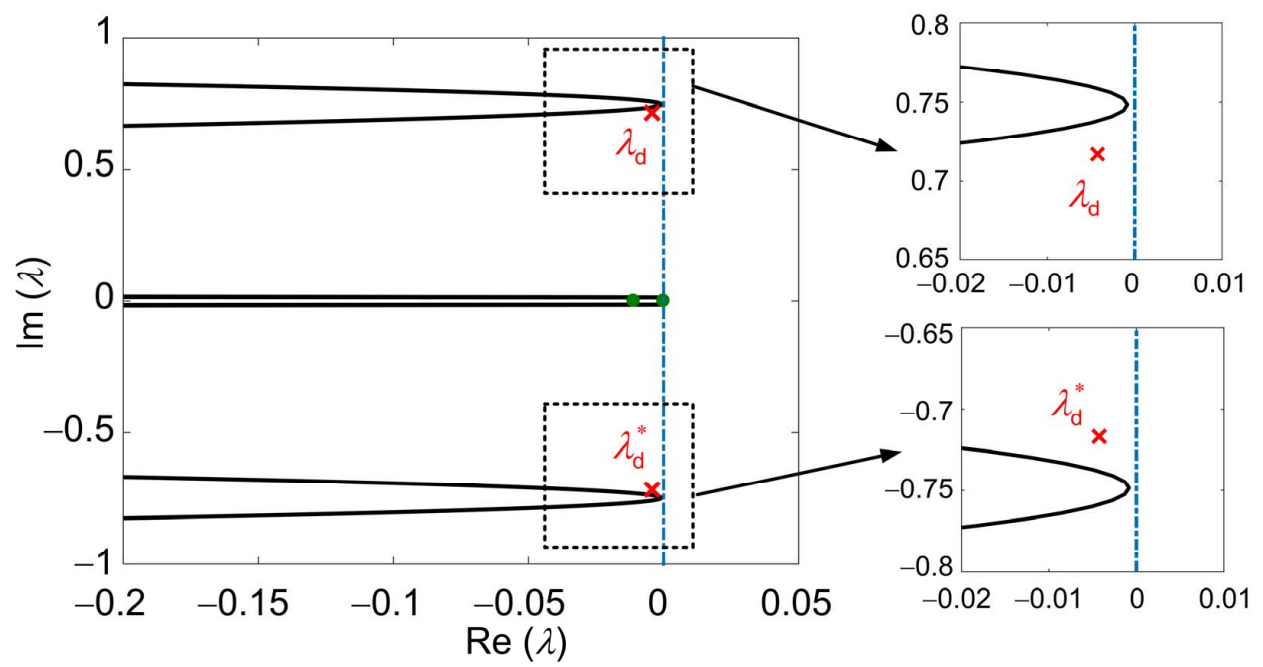

Fig. 4. The dynamical spectrum of $\mathrm{J}$ when $E_{\mathrm{sat}}=0.5, \alpha_{2}=23.76$, and $\alpha_{4}=-32.92$, corresponding to the single-channel modelocked solution that we show in Figs. 2(a) and 2(b).

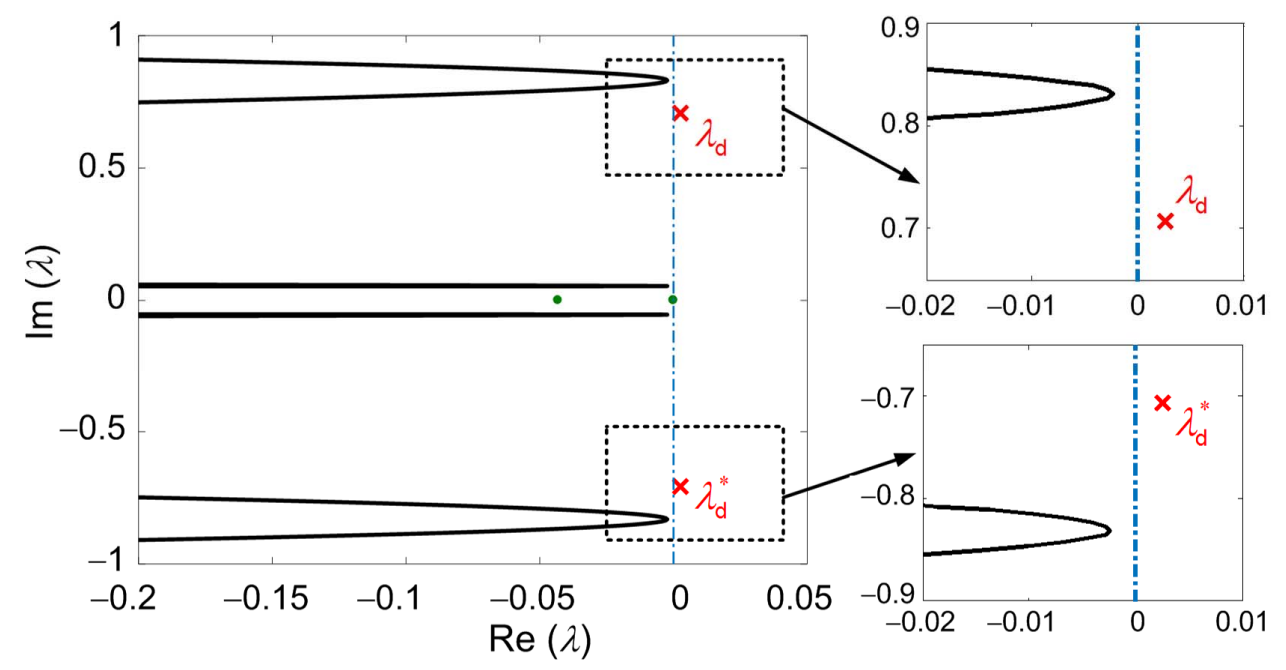

Fig. 5. The dynamical spectrum of $\mathrm{J}$ (for the single-channel modelocked solution) when $E_{\mathrm{sat}}=1.00, \alpha_{2}=23.76$, and $\alpha_{4}=-32.92$.

in Fig. 5. The real parts of the complex conjugate pair $\lambda_{\mathrm{d}}$ and $\lambda_{\mathrm{d}}^{*}$ becomes positive, which corresponds to a Hopf bifurcation [27,41].

In Fig. 6, we show the evolution of the unstable single channel modelocked solution when $E_{\text {sat }}=1.00$. As our initial condition, we use the single channel modelocked pulse when $E_{\mathrm{sat}}=0.5$, that we showed in Figs. 2(a) and 2(b). In the Fourier domain, we find that the optical energy in Ch-R increases, and eventually a new modelocked pulse forms. In the time domain, we observe a dual-channel modelocked state with a rapidly modulated amplitude. The dual-channel modelocked state that ultimately appears in Fig. 6 is identical to the state that we showed in 


\section{Optics EXPRESS}

Fig. 2(c).

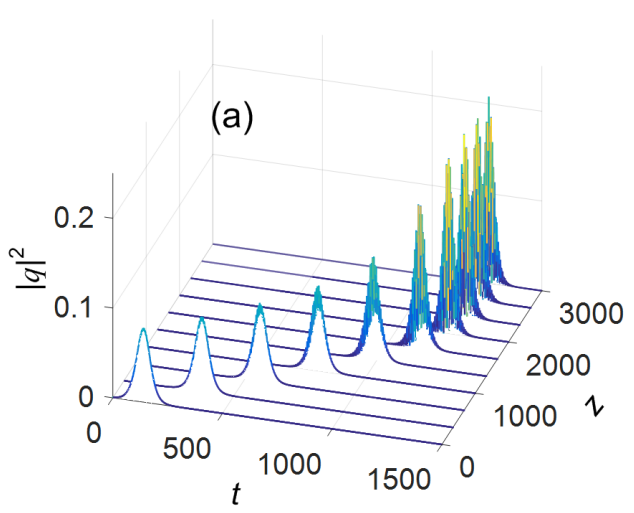

(b)

Fig. 6. Transients evolution from single pulse modelocking to dual-channel simultaneous modelocking in (a) the time and (b) the frequency domains.

However, as we have shown in Figs. 3(a) and 3(b), the dual-channel modelocked state is not observed when the channel separation, $\omega_{\mathrm{s}}$, becomes sufficiently large. Here, we consider the same set of parameters that are used in Fig. $3\left(E_{\mathrm{sat}}=2.5, \alpha_{2}=23.04\right.$, and $\left.\alpha_{4}=-17.98\right)$, corresponding to a filter channel separation of $\omega_{\mathrm{s}}=1.60$. By solving a root-finding problem, we obtain a single-channel modelocked stationary solution for these parameters, and we show its dynamical spectrum in Fig. 7. We observe that a portion of the continuous spectrum has positive real parts, which indicates that the single-channel stationary solution that we find is unstable.

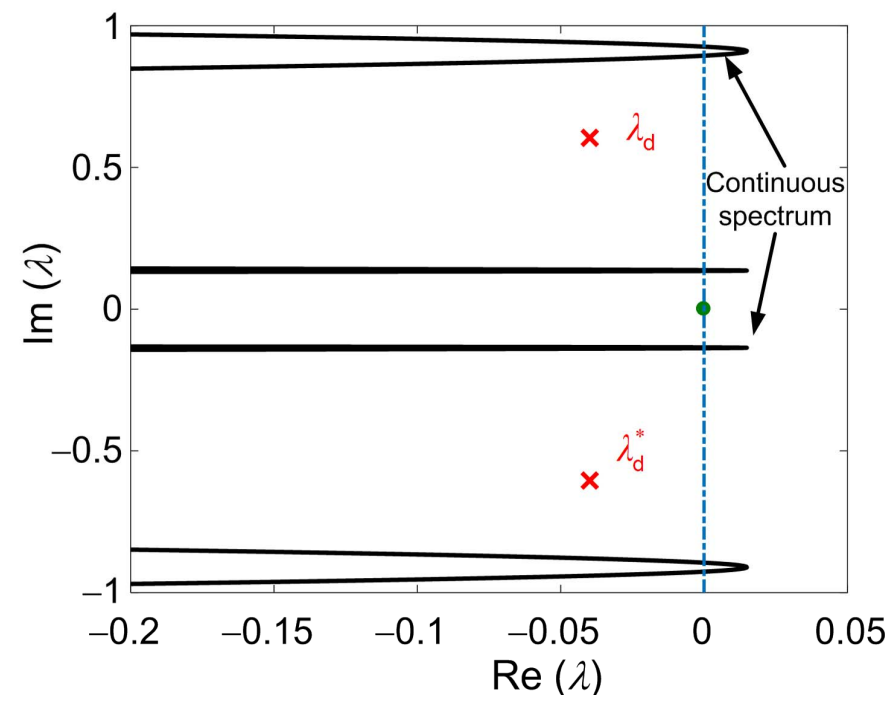

Fig. 7. The dynamical spectrum of the single channel modelocked pulse when $E_{\mathrm{sat}}=2.5$. The filter parameters are $\alpha_{2}=23.04, \alpha_{4}=-17.98$. The continuous spectrum crosses the imaginary axis, which indicates that a small perturbation that is separated from the modelocked pulse experiences net gain.

The continuous spectrum represents the case where $q(t) \approx 0$. We can write the Jacobian $\mathrm{J}$ in 
the Fourier domain as

$$
\mathrm{J}=\left[\begin{array}{cc}
i D_{1} \omega-\alpha_{2} \omega^{2}+\alpha_{4} \omega^{4}+\frac{g-l}{2} & \phi+\frac{\omega^{2}}{2} \\
-\phi-\frac{\omega^{2}}{2} & i D_{1} \omega-\alpha_{2} \omega^{2}+\alpha_{4} \omega^{4}+\frac{g-l}{2}
\end{array}\right],
$$

so that the eigenvalue $\lambda$ can be written as

$$
\lambda(\omega)=\frac{g-l}{2}-\alpha_{2} \omega^{2}+\alpha_{4} \omega^{4}+i D_{1} \omega \pm i\left(\phi+\frac{\omega^{2}}{2}\right) .
$$

The largest real parts of $\lambda(\omega)$ appears when $\omega= \pm \omega_{s} / 2$. Hence, the stability condition is

$$
\max [\operatorname{Re}(\lambda)]=\max \left[\frac{g-l_{\text {cavity }}}{2}+\alpha_{4}\left(\omega^{2}-\frac{\omega_{s}^{2}}{4}\right)^{2}\right]=\frac{g-l_{\text {cavity }}}{2}<0
$$

In order for the modelocked pulse to be stable, it follows that a small perturbation away from the modelocked pulse must experiences net loss. This result is consistent with prior work on multiple pulse modelocking dynamics [29,42-46]. Physically, the single channel modelocked pulse experiences the loss from the filter. When the pump power increases, the peak power of a modelocked pulse increases, and its Fourier spectrum is broadened due to the nonlinear Kerr effect, which then leads to a higher loss from the filter. However, the small signal is not affected by the filter and thus experiences an increased gain, which can grow up and become a modelocked pulse. Alternatively, changing other parameters will also lead to multi-pulsing, for example the group velocity dispersion [29], or saturable absorption [32], as they may lead to a small signal gain that exceeds the loss. In Fig. 8, we use the evolution approach to show this behavior in both the time domain and the frequency domain.
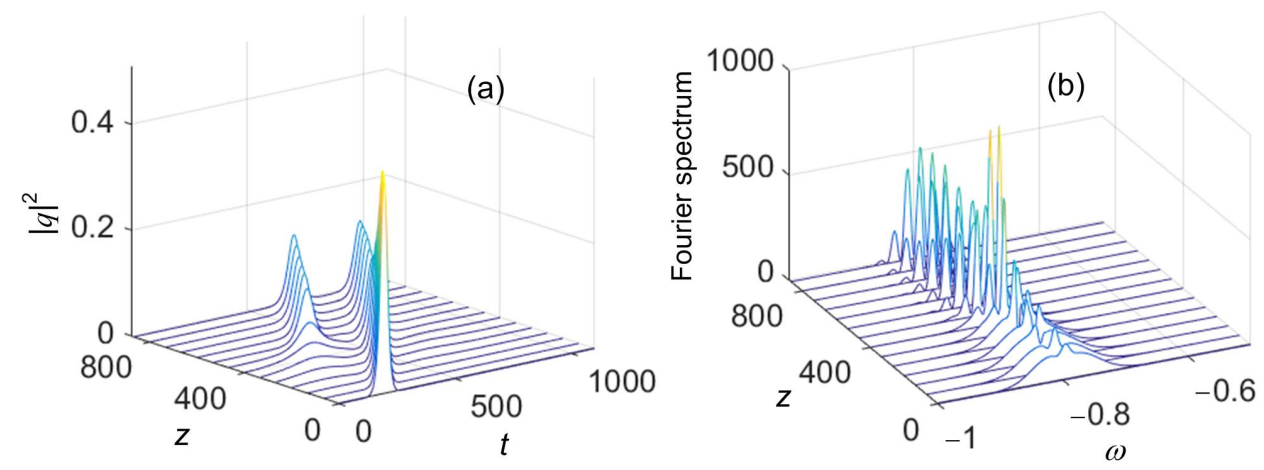

Fig. 8. Transition of a single-channel modelocked state to a multi-pulsing state (a) in the time and (b) in the frequency domain. We set $E_{\text {sat }}=2.5$. In the time domain, a new pulse appears, which leads to a rapid modulation in the frequency domain.

For a given filter channel bandwidth $\omega_{\mathrm{b}}$, increasing the pump power can destablize the single-channel modelocked state. The frequency separation of the filter channels, $\omega_{\mathrm{s}}$, determines whether the system evolves to a dual-channel modelocked state or a multi-pulsing state. When $\omega_{\mathrm{s}}$ is sufficiently small, the single-channel modelocking can evolve to dual-channel modelocked state. When $\omega_{\mathrm{s}}$ becomes sufficiently large and the cavity gain increases, the continuous spectrum has positive real parts, and the multi-pulsing instability occurs. In our model, we find that the 
threshold that divides the two states is $\omega_{\mathrm{s}}=1.54$, which, in physical units, corresponds to a threshold,

$$
\left|\Omega_{\mathrm{S}}\right|>1.54\left(\beta_{2} L_{\mathrm{c}}\right)^{-0.5}
$$

For example, when the group-velocity dispersion is $-20 \mathrm{ps}^{2} / \mathrm{km}$ and the cavity length is $L_{c}=10 \mathrm{~m}$, we can obtain a dual-channel simultaneous modelocked state when the separation of the two filter channels is less than $548 \mathrm{GHz}$.

\section{Conclusions}

Using the Swift-Hohenberg equation with a dual-channel filter, we study how to obtain a dualchannel modelocked state in a fiber laser. Depending on the cavity gain and the filter parameters, we can find single-channel modelocked states, dual-channel modelocked states, and multi-pulsing states. A single-channel modelocked state can evolve to a dual-channel modelocked state when the pump power increases. We obtain either a dual-channel modelocked state or a multi-pulse state, depending on the system parameters. Using the dynamical approach, we find that when the frequency separation of the filter channels is above a calculated threshold, the continuous eigenvalues become positive and lead to the multi-pulse instability. To obtain a dual-channel modelocked state, the frequency separation of the filter channels must be below the calculated threshold. The dynamical approach in this paper only applies to the Swift-Hohenberg equation. However, we can use it to any system of equations.

\section{Funding}

Hong Kong Research Grants Council (PolyU152144/15E); National Natural Science Foundation of China (61475131); Shenzhen Science and Technology Innovation Commission (JCYJ20160331141313917); Hong Kong Polytechnic University (1-ZVGB); Defense Advanced Research Projects Agency (DARPA) (W31P4Q-14-1-0002).

\section{References}

1. S. A. Diddams, “The evolving optical frequency comb,” J. Opt. Soc. Am. B 27, B51-B62 (2010).

2. S. Yun, C. Boudoux, M. Pierce, J. De Boer, G. Tearney, and B. Bouma, "Extended-cavity semiconductor wavelengthswept laser for biomedical imaging," IEEE Photonics Tech. L. 16, 293-295 (2004).

3. O. Kamatani, S. Kawanishi, and M. Saruwatari, "Prescaled $6.3 \mathrm{ghz}$ clock recovery from $50 \mathrm{gbit} / \mathrm{s} \mathrm{tdm}$ optical signal with $50 \mathrm{ghz}$ pll using four-wave mixing in a travelling-wave laser diode optical amplifier," Electron. Lett. 30, 807-809 (1994).

4. M. A. Solodyankin, E. D. Obraztsova, A. S. Lobach, A. I. Chernov, A. V. Tausenev, V. I. Konov, and E. M. Dianov, "Mode-locked $1.93 \mu \mathrm{m}$ thulium fiber laser with a carbon nanotube absorber," Opt. Lett. 33, 1336-1338 (2008).

5. F. Bonaccorso, Z. Sun, T. Hasan, and A. C. Ferrari, "Graphene photonics and optoelectronics," Nat. Photonics 4 , 611-622 (2010).

6. X. Liu, "Interaction and motion of solitons in passively-mode-locked fiber lasers," Phys. Rev. A 84, 053828 (2011).

7. K. Kieu and F. W. Wise, "All-fiber normal-dispersion femtosecond laser," Opt. Express 16, 11453-11458 (2008).

8. Z. Sun, T. Hasan, F. Wang, A. G. Rozhin, I. H. White, and A. C. Ferrari, "Ultrafast stretched-pulse fiber laser mode-locked by carbon nanotubes," Nano Res. 3, 404-411 (2010).

9. V. J. Matsas, T. P. Newson, D. J. Richardson, and D. N. Payne, "Selfstarting passively mode-locked fibre ring soliton laser exploiting nonlinear polarisation rotation," Electron. Lett. 28, 1391-1393 (1992).

10. C.-J. Chen, P. K. A. Wai, and C. R. Menyuk, "Soliton fiber ring laser,” Opt. Lett. 17, 417-419 (1992).

11. Z. C. Luo, A. P. Luo, W. C. Xu, H. S. Yin, J. R. Liu, Q. Ye, and Z. J. Fang, "Tunable multiwavelength passively mode-locked fiber ring laser using intracavity birefringence-induced comb filter," IEEE Photonics J. 2, 571-577 (2010).

12. U. Keller, K. J. Weingarten, F. X. Kartner, D. Kopf, B. Braun, I. D. Jung, R. Fluck, C. Honninger, N. Matuschek, and J. A. der Au, "Semiconductor saturable absorber mirrors (sesam's) for femtosecond to nanosecond pulse generation in solid-state lasers," IEEE J. Sel. Top. Quantum Electron. 2, 435-453 (1996).

13. H. Zhang, D. Y. Tang, X. Wu, and L. M. Zhao, "Multi-wavelength dissipative soliton operation of an erbium-doped fiber laser," Opt. Express 17, 12692-12697 (2009).

14. X.-M. Tan, H.-J. Chen, H. Cui, Y.-K. Lv, G.-K. Zhao, Z.-C. Luo, A.-P. Luo, and W.-C. Xu, "Tunable and switchable dual-waveband ultrafast fiber laser with 100 ghz repetition-rate," Opt. Express 25, 16291-16299 (2017). 
15. E. D. Farnum, L. Butson, and J. N. Kutz, "Theory and simulation of dual-frequency mode-locked lasers," J. Opt. Soc. Am. B 23, 257-264 (2006).

16. E. D. Farnum and J. N. Kutz, "Multifrequency mode-locked lasers," J. Opt. Soc. Am. B 25, 1002-1010 (2008).

17. G. Hu, Y. Pan, X. Zhao, S. Yin, M. Zhang, and Z. Zheng, "Asynchronous and synchronous dual-wavelength pulse generation in a passively mode-locked fiber laser with a mode-locker," Opt. Lett. 42, 4942-4945 (2017).

18. X. Zhao, Z. Zheng, L. Liu, Y. Liu, Y. Jiang, X. Yang, and J. Zhu, "Switchable, dual-wavelength passively mode-locked ultrafast fiber laser based on a single-wall carbon nanotube modelocker and intracavity loss tuning," Opt. Express 19, 1168-1173 (2011).

19. R. Li, H. Shi, H. Tian, Y. Li, B. Liu, Y. Song, and M. Hu, "All-polarization-maintaining dual-wavelength mode-locked fiber laser based on sagnac loop filter," Opt. Express 26, 28302-28311 (2018).

20. J. Lhermite, D. Sabourdy, A. Desfarges-Berthelemot, V. Kermene, A. Barthelemy, and J.-L. Oudar, "Tunable high-repetition-rate fiber laser for the generation of pulse trains and packets," Opt. Lett. 32, 1734-1736 (2007).

21. J. Schröder, D. Alasia, T. Sylvestre, and S. Coen, "Dynamics of an ultrahigh-repetition-rate passively mode-locked raman fiber laser," J. Opt. Soc. Am. B 25, 1178-1186 (2008).

22. A. Pasquazi, M. Peccianti, B. E. Little, S. T. Chu, D. J. Moss, and R. Morandotti, "Stable, dual mode, high repetition rate mode-locked laser based on a microring resonator," Opt. Express 20, 27355-27363 (2012).

23. M. Quiroga-Teixeiro, C. B. Clausen, M. P. Sørensen, P. L. Christiansen, and P. A. Andrekson, "Passive mode locking by dissipative four-wave mixing," J. Opt. Soc. Am. B 15, 1315-1321 (1998).

24. T. Sylvestre, S. Coen, O. Deparis, P. Emplit, and M. Haelterman, "Demonstration of passive modelocking through dissipative four-wave mixing in fibre laser," Electron. Lett. 37, 881-882 (2001).

25. J. M. Soto-Crespo and N. Akhmediev, "Composite solitons and two-pulse generation in passively mode-locked lasers modeled by the complex quintic swift-hohenberg equation," Phys. Rev. E 66, 066610 (2002).

26. B. Deconinck and J. N. Kutz, "Computing spectra of linear operators using the Floquet Fourier Hill method," J. Comput. Phys. 219, 296-321 (2006).

27. S. Wang, A. Docherty, B. S. Marks, and C. R. Menyuk, "Boundary tracking algorithms for determining the stability of mode-locked pulses," J. Opt. Soc. Am. B 31, 2914-2930 (2014).

28. S. Wang and C. Menyuk, "Computational methods for determining the stability of pulses in passively modelocked laser systems," in 2013 IEEE Photonics Conference (IPC), (2013), pp. 392-393.

29. M. J. Lederer, B. Luther-Davies, H. H. Tan, C. Jagadish, N. N. Akhmediev, and J. M. Soto-Crespo, "Multipulse operation of a ti:sapphire laser mode locked by an ion-implanted semiconductor saturable-absorber mirror," J. Opt. Soc. Am. B 16, 895-904 (1999).

30. H. A. Haus, "Theory of mode locking with a fast saturable absorber," J. Appl. Phys. 46, 3049-3058 (1975).

31. F. Kärtner, I. Jung, and U. Keller, "Soliton mode-locking with saturable absorbers," IEEE J. Sel. Top. Quantum Electron. 2, 540-556 (1996).

32. F. X. Kärtner, J. A. der Au, and U. Keller, "Mode-locking with slow and fast saturable absorbers-what's the difference?" IEEE J. Sel. Top. Quantum Electron. 4, 159-168 (1998).

33. B. G. Bale, E. Farnum, and J. N. Kutz, "Theory and simulation of passive multifrequency mode-locking with waveguide arrays," IEEE J. Quantum Elect. 44, 976-983 (2008).

34. X. Zhang, S. Wang, F. Li, C. R. Menyuk, and P. Wai, "Design of a dual-channel modelocked fiber laser that avoids multi-pulsing," in CLEO: Applications and Technology, (Optical Society of America, 2018), pp. JTh2A-125.

35. N. Akhmediev and A. Ankiewicz, Dissipative Solitons in the Complex Ginzburg-Landau and Swift-Hohenberg Equations (Springer Berlin Heidelberg, Berlin, Heidelberg, 2005).

36. S. Wang, A. Docherty, B. S. Marks, and C. R. Menyuk, "Comparison of numerical methods for modeling laser mode locking with saturable gain,” J. Opt. Soc. Am. B 30, 3064-3074 (2013).

37. C. R. Menyuk and S. Wang, "Spectral methods for determining the stability and noise performance of passively modelocked lasers," Nanophotonics 5, 332-350 (2016).

38. S. Wang, S. Droste, L. C. Sinclair, I. Coddington, N. R. Newbury, T. F. Carruthers, and C. R. Menyuk, "Wake mode sidebands and instability in mode-locked lasers with slow saturable absorbers," Opt. Lett. 42, 2362-2365 (2017)

39. H. A. Haus and A. Mecozzi, "Noise of mode-locked lasers," IEEE J. Quantum Elect. 29, 983-996 (1993).

40. M. J. Ablowitz and A. S. Fokas, Complex variables: introduction and applications (Cambridge University, 2003).

41. S. Strogatz, Nonlinear Dynamics and Chaos: With Applications to Physics, Biology, Chemistry and Engineering, Studies in Nonlinearity Series (Perseus Books Publishing, 1994).

42. F. Li, P. K. A. Wai, and J. N. Kutz, "Geometrical description of the onset of multi-pulsing in mode-locked laser cavities,” J. Opt. Soc. Am. B 27, 2068-2077 (2010).

43. F. Li, E. Ding, J. N. Kutz, and P. K. A. Wai, "Dual transmission filters for enhanced energy in mode-locked fiber lasers," Opt. Express 19, 23408-23419 (2011).

44. D. Li, D. Tang, L. Zhao, and D. Shen, "Mechanism of dissipative-soliton-resonance generation in passively mode-locked all-normal-dispersion fiber lasers," J. Light. Technol. 33, 3781-3787 (2015).

45. X. Zhang, F. Li, Z. Kang, J. Yuan, and P. K. A. Wai, "Spectral filtering induced multi-pulsing in mode-locked soliton lasers," in Photonics and Fiber Technology 2016 (ACOFT, BGPP, NP), (Optical Society of America, 2016), p. JT4A.7.

46. X. Zhang, F. Li, K. Nakkeeran, J. Yuan, Z. Kang, J. N. Kutz, and P. K. A. Wai, "Impact of spectral filtering on multipulsing instability in mode-locked fiber lasers," IEEE J. Sel. Top. Quantum Electron. 24, 1-9 (2018). 